\title{
INTERDISCIPLINARY MEDICAL AND DENTAL DESENSITIZATION FOR PEOPLE WITH AUTISM
}

\author{
AUTHORS' DATA \\ Victoria Babikian, MS, OTR/L \\ Premier HealthCare, USA \\ Occupational Therapist, MS in Occupational Therapy, OTR/L \\ Contacts: Victoria.Babikian@yai.org \\ Lena Kadota, MS, OTR/L \\ Premier HealthCare, USA \\ Occupational Therapist, MS in Occupational Therapy, OTR/L \\ Contacts: Lena.Kadota@yai.org
}

Allison Valeriano, MS, OTR/L

Premier HealthCare, USA

Occupational Therapy Supervisor, MS in Occupational Therapy, OTR/L

Contacts: Allison.Valeriano@yai.org

\begin{abstract}
Premier HealthCare, a member of the YAI Network, specializes in outpatient healthcare services (such as primary care, dentistry, psychiatry, rehabilitation, psychotherapy, psychological testing, and nutrition) for people with intellectual and developmental disabilities in New York City. This is one of the most in need, yet underserved, groups of medical and dental patients. Many people with autism spectrum disorder (ASD) have difficulty understanding the significance of healthcare procedures and become overwhelmed by the unfamiliar environment and sensory input of medical and dental practices. They may also have difficulty with communication and socialization, which can exacerbate anxiety and challenging behaviors such as self-stimulation, self-injury, aggression, and tantrums. Additionally, this heightened level of stress can potentially translate into disruptive behaviors or physical reactions to avoid treatment. These challenges
\end{abstract}


combined with a lack of healthcare professionals trained and experienced in working with people with ASD can result in ineffective preventative care and high-cost procedures.

At Premier HealthCare, a desensitization program was initiated to increase functional participation in medical and dental exams and decrease use of physical restraints or general anesthesia for people with ASD. An interdisciplinary team of occupational therapists, behavior analysts, doctors, nurses, and dentists collaborate to address how to make medical and dental procedures more tolerable for patients with special needs using a protocol comprising of four main stages: evaluation, treatment, generalization, and maintenance. They work with caregivers to provide person-centered individualized care, ultimately leading to decreased anxiety, fear, and aggressive behaviors; increased participation; and better health outcomes for people with autism.

Keywords: Occupational therapy; disability; pediatrics; primary care; intellectual disability; developmental disability; desensitization; interdisciplinary; autism; dental desensitization; medical desensitization.

\section{INTRODUCTION}

The YAI network is a nonprofit organization that provides innovative services for people with intellectual and developmental disorders (I/DD). The organization promotes a "total life adjustment approach, emphasizing personal growth, social responsibility, employment goals, and the development of independence for the individual" (YAI, n.d.). Premier HealthCare is a member agency of the YAI network. It consists of a group of outpatient clinics that provide medical, dental, psychiatry, rehabilitation, mental health, and nutrition services primarily for the I/DD community throughout New York City. It is recognized as a Patient-Centered Medical Home by the National Committee on Quality Assurance (YAI, n.d.).

A desensitization program at Premier, funded by a grant from the New York State Office for People with Developmental Disabilities (OPWDD) was initiated to enhance functional participation during medical and dental exams. Desensitization is a type of behavioral therapy used by trained professionals to help people overcome fears, phobias and other anxiety disorders. It teaches relaxation and stress management techniques, while gradually exposing people to distressing situations, so the person can eventually face the situation without fear or anxiety (Davit, Hundley, Bacic \& Hanson, 2011). At Premier, occupational therapists, behavioral analysts, nurses, 
doctors, dentists, and dental/medical assistants collaborate to address the anxiety, fears, and aggressive behaviors that can disrupt routine health management.

The American Occupation Therapy Association recognizes health care management as an instrumental activity of daily living (IADL) that supports daily life within the home and community. It involves developing, managing, and maintaining routines for health and wellness promotion (AOTA, 2014). Neuro typical individuals can struggle with this IADL due to fear, anxiety, lack of access to healthcare, and/or a decreased understanding of the importance of health maintenance and wellbeing. Individuals with disabilities are at an even greater disadvantage to participate in health care management due to developmental deficits, co-morbidities, and systemic barriers.

Research shows that people with autism spectrum disorder (ASD) are less likely to utilize preventative services, have more frequent visits to emergency departments, and have an increased number of unmet health needs compared to non-autistic adults (Nicolaidis, et al., 2015; Schell, Gillen \& Scaffa, 2014). ASD is also associated with increased prevalence of several medical conditions such as immune conditions, gastrointestinal disorders, sleep disorders, seizure, obesity, dyslipidemia, hypertension, and diabetes contributing to an increased mortality risk (Croen et al. 2015; Hirvikoski et al. 2016). As a result, adults with ASD have poorer health and decreased lifeexpectancy than the general adult population (Mouridsen, Brønnum-Hansen, Rich \& Isager, 2008). Thus, health care management is a crucial and life-saving activity for this population.

\section{LITERATURE REVIEW}

As specified in the diagnosis name, people with ASD present with a wide range of symptoms, skills, and levels of disability. Within this diversity there are several common features of ASD that can become barriers to participating in health care services such as social impairment, communication difficulties, and difficulty adapting to changes in routine.

Cognitive and communication differences can complicate identification and management of illnesses unrelated to the disability (Nicolaidis, Kripke \& Raymaker, 2014). Many individuals with autism are unable to identify and communicate health changes to their caregivers. Research highlights the possibility that people with autism may not express physical discomfort in the same way as a neurotypical individual. This may lead caregivers and clinicians to interpret this as pain insensitivity or incorrectly lead them to believe that there is no pain - further complicating the 
assessment of their health status (Allely, 2013). Communication differences can also impair their ability to describe symptoms, follow directions, and understand the purpose of the doctors' actions causing anxiety and resulting in disruptive behaviors to avoid health management.

Several studies have shown that individuals with autism present with significantly greater levels of anxiety and anxiety disorders when compared to age-matched peers (Kim, Szatmar, Bryson, Streiner \& Wilson, 2000; Ferrugia \& Hudson, 2006; Evans, Canavera, Kleinpeter, Maccubbin \& Taga, 2005). Fear and anxiety present as an obstacle to health care management in several ways. Firstly, individuals who fear medical and dental procedures are much more likely to delay or avoid exams and procedures, as well as regularly cancel or fail to keep appointments (Armfield, 2012). Secondly, fearful patients may prove difficult to treat, require more time, and present with behavioral problems resulting in a stressful experience for both patient and practitioner. A study conducted by Brahm, Lundgren, Carlsson, Nilsson, Corbeil and Hagglin (2012) indicates that caring for patients with dental fear is a source of considerable stress for many dentists and is associated with hard work, poor revenues, and little appreciation by employers. Finally, due to avoidance of preventative care, fearful patients often have poorer dental health. Those who delay dental care for a prolonged time might have extensive problems that require more complex and involved treatment (Armfield \& Heaton, 2013).

Many people with I/DD have difficulty understanding and anticipating the social situations and contexts that occur in medical and dental procedures. Medical clinics and hospitals are dynamic environments where unexpected changes and uncertainty are common. This can be challenging for an individual who struggles with flexibility and deviations from routine. Within the appointments themselves, medical professionals use instruments and tools that may seem menacing, and the procedures can appear frightening or painful ultimately provoking a fight-orflight response (Hawkins, 1991).

Sensory processing deficits emerge at a young age and are so commonly seen in people with autism that it can be considered universal feature of ASD and a potential diagnostic criterion (Thye, Bednarz, Herringshaw, Sartin \& Kana, 2017; Tomchek \& Dunn, 2007). Sensory processing is defined as the effective registration and accurate interpretation of sensory input from the environment. Sensory modulation is defined as the capacity to regulate and organize the information in order to remain at an appropriate level of alertness to adapt to challenges in daily life. Many people with ASD demonstrate difficulty filtering and developing an adaptive response 
to sensory stimuli (Tomchek \& Dunn, 2007). The volume of environmental and novel/unfamiliar stimuli experienced in a medical setting can be overwhelming for individuals with sensory processing disorders. In an effort to regulate their systems individual may attempt to escape to an area with less stimulation or engage in self-stimulatory or self-injurious behaviors.

These challenging responses, coupled with a lack of health care professionals trained and experienced in working with this population, result in insufficient access to care and poor health. Most primary care providers lack the necessary training to care for patients with autism (Zerbo, Massolo, Qian \& Croen, 2015). Medical and dental providers may not know how to interpret some of their patients' behaviors and make incorrect assumptions about their abilities and needs (Nicolaidis et. al., 2015). Providers may not be able to adapt to the situation appropriately and as a result opt to have patients physically restrained (strapped or held down) or sedated for routine procedures. Parents have expressed a fear or discomfort with these physical and chemical restraints as these can cause lasting negative associations and responses. These negative experiences may cause emotional distress and aversion to participation in future unrelated health appointments.

In addition, anesthesia and physical restraints can negatively impact the body. Procedural sedation side effects may have deleterious effects on the patient's physical and emotional wellbeing. Possible side effects are change in heart rate and blood pressure, decreased rate of breathing, headache, inhalation of stomach contents into the lungs, nausea and vomiting, disorientation, and confusion (Johns Hopkins Medicine, n.d.).

Physical restraint can be dangerous as it involves physical struggle, discomfort, and it may put pressure on the chest or interrupt breathing. In addition, it has been postulated that potentially fatal cardiac arrhythmia can result from the combination of certain medications and the adrenaline produced by an individual's agitation during restraint procedures (Putallaz, Rubins \& Schwartz, 1999). The patient is not the only one at risk during physical restraint as health care workers may also be injured while attempting to restrict a struggling patient's movements. Participating in routine medical and dental procedures without the use of a restraint is beneficial not only to the patient and family but also to the health care professionals involved.

Desensitization programs aim to increase the voluntary participation in routine medical procedures by reducing patient anxiety surrounding medical and dental procedures. This is done by empowering the patient with knowledge of what to expect through gradual exposure to the 
aspects of the procedure that cause anxiety. Research shows that desensitization techniques can increase compliance with dental and medical procedures in people with autism (Nelson, Chim, Sheller, McKinney \& Scott, 2017; Kuhaneck, \& Chisholm, 2012; Primeau, Gershon \& Talbot, 2016; Davit, Hundley, Bacic \& Hanson, 2011).

With a collaborative approach involving sensory integration, neuromuscular re-education, therapeutic exercises, behavior analysis, environmental modification, and co-treatment the desensitization program at Premier aims to address the challenges faced by patients with ASD and caregivers during routine medical and dental examinations. This team strives to decrease the use of physical restraints and general anesthesia, help promote health management, and ultimately enhance wellbeing for individuals with autism.

\section{METHODOLOGY}

\section{The Team}

What makes the desensitization program at Premier HealthCare unique is the collaborative co-treatment approach designed to foster dialogue between diverse disciplines and promote the development of an integrated health care model. Members of the team include the patient, their family/support system, the occupational therapist (OT), behavior analyst, as well as the dental, medical, and nursing staff.

Participants in the grant must meet the following criteria:

- OPWDD eligibility (disability meets the requirements specified in New York State) (OPWDD, n.d.)

- Autism spectrum disorder diagnosis

- Live with family

- Live in Queens or Brooklyn regions of New York City

- Have displayed challenging behaviors in previous attempts at dental or medical treatment (i.e. refusing to enter the dental/medical room, crying, screaming, rocking, and acting aggressively towards self and/or others)

- Due to the extent of these behaviors, more extreme measures may have previously been necessary (stabilization devices or general anesthesia) to administer critical medical and/or dental care. 
The desensitization intervention is patient-centered and inclusive of the person's family or caregiver support system. It is important to talk to the patient and their family about their likes, dislikes, and interests to build rapport and for carryover of skills learned. Often, the therapists will provide tools or model strategies that the family can continue to practice at home in order to promote regular simulations of sensations and events that can take place during a routine exam (i.e. plastic tools to familiarize with the process, tactile input to decrease hypersensitivity, etc.).

Occupational therapy practitioners utilize a holistic model by assessing sensory processing, as well as the impact of the environment and procedures on the individual. OT helps individuals across the lifespan participate in the activities they want and need to do through the therapeutic use of everyday activities. In this program, the highlighted activity is participating in routine medical and dental exams, or health care management. With their knowledge of sensory integration, ergonomics, and environmental impacts, the OT works with the team to identify accommodations or modifications to the environment and procedures. In addition, the OT serves as a liaison between the patient, caregiver, and the rest of the desensitization team.

The board-certified behavior analyst plays an important role in helping patients learn the skills needed for success by systematically measuring changes in behavior in relation to events that come before (antecedent) and after (consequence) the behavior of interest (completing medical/dental procedure). Some of the principles underlying human behavior include reinforcement (functional relationship between two events that increase behavior) and punishment (functional relationship between two events that decrease behavior). Individuals who are fearful of medical and dental procedures often have negative associations from previous experiences. The behavior analyst works with the patient and caregiver to identify meaningful rewards in order to positively reinforce participation in appointments and reshape the patient's view of medical and dental procedures. Additionally, their ability to analyze the context in which behaviors present is vital in making modifications and accommodations to the environment and procedure.

Of course, the central part of this program and the ultimate goal is for patients to complete their regular health care visits with the medical, nursing, and dental staff. The relationship between doctor and patient is key to the success of desensitization program. Trust in specific doctors and nurses allows the patient to have positive associations with medical/dental procedures and furthers the process toward generalization. 
The Process

The desensitization program has 4 major stages: evaluation, intervention, generalization, and maintenance.

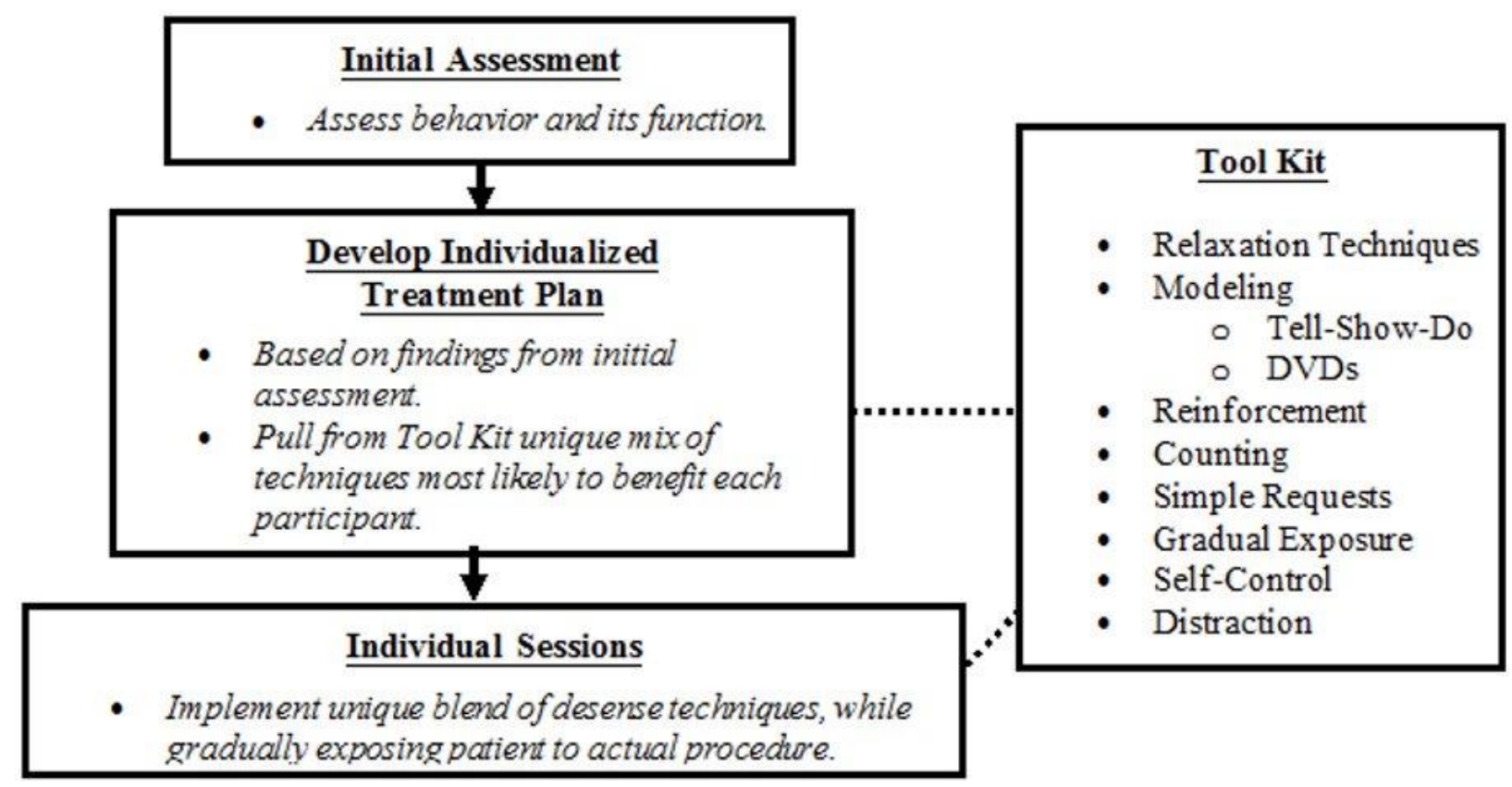

The purpose of the evaluation phase is to identify the target behavior for intervention, determine the function of the behavior, and establish a baseline. The occupational therapist and behavior analyst assess the patient's skills, challenges, behaviors, and their functions. The OT assesses skills including cognition, visual perception, sensory processing, sensation, motor coordination, endurance, tone, functional balance, self-care, etc. The behavior analyst's evaluation includes a daily activity log, communication profile, behavioral language assessment, preference assessment, life activities checklist, and functional behavioral assessment. Information is gathered to assess the person's past experiences with medical/dental services, aspects that provoke the most anxiety, degree of fear surrounding different components of the exam, and if any calming techniques were developed from previously stressful situations.

Based on these results, an individualized treatment plan is developed including the target behavior, goals for intervention, and desensitization techniques that would likely meet the unique needs of the patient to reduce their anxiety. Below are some examples of occupational therapy and behavioral goals: 
- Occupational Therapy Goal: Joe will demonstrate improved sensory processing/modulation of oral-tactile, vestibular, and proprioceptive input as evidenced by tolerating simulated teeth cleaning with prophy and hand scaling for 20 minutes with moderate verbal cues for direction-following to increase performance in dental health management.

- Behavioral Goal: Joe will display his ability to comfortably complete the prerequisite skills (i.e., communicate his needs, follow directives) as needed to successfully engage in medical/dental procedures; without excessive avoidant behavior (i.e. persistent vocal/verbal/physical resistance, escape).

OTs can incorporate sensory strategies to help patients stay calm and self-regulate in order to participate in their sessions. Most people are not aware of the volume of sensory stimuli present during health care appointments and there are many aspects of a medical/dental visit that may cause sensory overload for people with autism. People with tactile (touch) defensiveness may be sensitive to the feeling of probing hands, needles, bandages, thermometers, dental tools, vibrations in the mouth, and blood pressure cuffs. Those with olfactory and oral deficits may experience aversion to strong smells and/or tastes such as prophy paste, rinses, alcohol swabs, and cleaning solutions. Auditory system deficits may produce difficulty tolerating sounds of motorized dental tools and crowded waiting areas. Those with visual hypersensitivity can be distressed by bright lights (dental light, fluorescent lights in the clinic, and ophthalmoscopes for eye exams).

Touch, smell, taste, and sound are the more obvious stimuli in a medical clinic, however those with difficulty processing movement and pressure can also experience challenges during these appointments. Individuals with a disrupted vestibular system may dislike ordinary physical movements and experience motion sickness from riding in cars, elevators, or escalators making transportation to and from appointments difficult. In the case of gravitational insecurity, individuals may experience anxiety when their feet leave the ground, fear falling off on a high exam tables and dental chairs, or have poor tolerance of reclined exam chairs. In contrast, those who seek intense movement may have trouble sitting in a waiting room or remaining still for an exam (this is particularly important during blood draws, blood pressure readings, and dental work) and may attempt to elope. Lastly, proprioceptive system deficits may present as self-injurious behavior such as hitting or biting themselves and chewing or mouthing tools. The occupational therapist may incorporate sensory strategies during simulated medical visits which include using 
a weighted lap pad for calming deep pressure (the dental x-ray vest is a good alternative), headphones to muffle sounds, fidget objects to squeeze, oral motor chew toys, etc.

Outside of sensory processing issues, there are other challenges that may make healthcare participation difficult. Individuals with ASD often have low muscle tone or generalized weakness which results in difficulty maintaining open mouth position during dental procedures or inability to remain in certain positions necessary for medical procedures such as sitting unsupported during chest exam or during blood pressure readings. Therefore, the OT may also address muscle strength during intervention or utilize adaptive equipment for positioning in order to enhance participation during routine medical and dental visits.

Behavioral strategies may include modeling (the demonstration of a behavior), shaping (use of differential reinforcement to teach/learn new behavior), fading (the temporary use of a prompt or cue, errorless learning/teaching, and questions about the observed excessive response (a patient demonstrating excessive echolalia may be asked "Are you talking about something that happened in school?'). The stimuli that triggers or eases a patient's excessive response and what was shown to be effective and/or ineffective in each session is analyzed.

The OT is trained by the medical and dental staff on accurate procedure and tool use, which allows for simulations during intervention. The OT and behavior analyst utilize co-treatment desensitization techniques, sensory integration, and behavioral strategies during simulated procedures. These simulations occur in the exam rooms with the actual tools used by the dental providers (i.e. dental mirror, explorer, air water syringe, saliva ejector, prophy handpiece, etc.) and medical providers (i.e. thermometer, sphygmomanometers, tourniquet, syringe, etc.).

The patient is gradually taken through the steps in the procedural sequence with individualized interventions. The patient is never forced to complete a step as it is counterproductive to the process. Every opportunity is given to provide choices (i.e. which exam room, which arm, etc.), explanations of tools/procedures, time to calm with sensory strategies as needed if agitated, and positive reinforcement throughout the session. The therapist makes every attempt to make sessions enjoyable for the patient and will end each session on a good note. It is important to know that the speed of the process is directed by the patient and may take many months or years. The team uses a gradual approach to achieve a "just right" challenge.

The primary area of concern for medical visits with these patients is their fear of needles. Treatment may first address simply getting a patient comfortable sitting in the exam room while 
engaged in a preferred activity, such as playing games, for a positive association. Once comfortable, preparatory steps to the procedure can be introduced (i.e. rolling up their sleeve, tying the tourniquet, wiping the area with an alcohol pad). This can be done with demonstration or even role reversal (patient acting as the medical provider while the therapist acts as the patient) in order to decrease the fear associated with these procedures. Eventually a capped needle may be introduced touching it on their fingertips, then gradually moving up their arm, and so on, until the procedure is tolerated.

A similar approach can be used for dental visits by first acclimating patients to the dental room environment, including remaining seated in the exam chair. The next step may be demonstration of how the dental tools look and sound like before applying tools distally (i.e. fingertips) and gradually moving up towards the lips/mouth. Colorful plastic tools may be used first and can be less intimidating than the metal tools that are primarily used.

Modifications can also be made to the environment or activity in order to adapt to the needs of each patient. Environmental modifications can include decreased visual stimuli (i.e. dimming the lights of the room, using sunglasses), decreased auditory stimuli (i.e. using noise-canceling headphones, playing calming music), and removal of triggers (i.e. provider not wearing the white coats as this may be intimidating). Activity modifications can include use of a visual schedule or social story to outline anticipated steps, incorporation of sensory strategies and breaks as needed, use of a token board or reinforcers as a reward system, and the therapist joining the patient in their actual medical/dental appointment for additional support and facilitation of strategies with the provider.

Caregivers should be educated on strategies and tools to help carryover the skills learned and to apply to their real medical and dental appointments (especially if they are outside of Premier). They can be given typical items used in medical and dental procedures to role-play going to the dentist/doctor at home (e.g. face masks, gloves, non-invasive tools such as the plastic tools, simulations). Social stories using photos or videos (real-life, animated, or of the patients/staff themselves) can be reviewed to explain the steps involved in their specific appointment.

In the generalization phase, the patients apply these strategies and skills to their real medical and dental appointments. This happens when the patient is ready to transfer their acquired skills to work with unfamiliar health care providers. In maintenance, patients have periodic therapy sessions to ensure they continue to have success in their appointments. The patient is seen on an 
as-needed basis in order to ensure preservation of learned skills and prevention of regression. While not all patients will complete every aspect of their medical and dental visits, this team strives for success by having patients be able to tolerate more than they could before without use of restraints and with a more positive overall experience.

\section{$\underline{\text { Data Collection }}$}

Data is obtained for each session through progress note narratives and the Procedural Resistance Scale (PRS), the outcome measure form developed by Premier's desensitization team at the inception of the program. The PRS includes basic information about the type of visit or procedures done, a breakdown of the steps of the medical or dental visit, a scale to indicate level of resistance observed during each step, behaviors exhibited by the patient, interventions used, a Likert scale to assess level of improvement from previous sessions, and a comment section for any additional pertinent information. It must be noted that this is not a standardized assessment tool, but a form of documentation that was created specifically for this program.

\section{DISCUSSION}

Coordination of care is essential when utilizing various health care services for the individual to have the highest benefit. Effective teams are characterized by communication and collaboration. There are various models of interdisciplinary teamwork within healthcare: multidisciplinary, interdisciplinary, transdisciplinary. Multidisciplinary teams are characterized by various disciplines working together under one roof, each completing their own responsibilities with the patient at the epicenter. This best describes Premier HealthCare as a whole-providing various services to patients, each with distinctive patient-centered goals and treatment plans (Schell, Gillen \& Scaffa, 2014).

Contrarily, interdisciplinary teams work together with the client to determine goals, how each team member will contribute, and create a collaborative plan. This team best describes the desensitization grant, as some interventions may be jointly carried out and the client is involved in the decision-making process. There can be issues that arise from an interdisciplinary team, however, if there is an uneven distribution of responsibility between team members. One team member may take the lead, pushing their own agenda further than another team member. For this 
reason, communication and trust are essential for the desensitization program to be a success (Schell, Gillen \& Scaffa, 2014).

Transdisciplinary teams have interchangeable roles; expertise and responsibilities are shared. This is evident in the occupational therapist's approach as they incorporate aspects of behavior management, dental/medical intervention, and their own occupational therapy strategies throughout the desensitization process. It is important to note that frequent consultation with other disciplines is necessary to ensure accurate implementation of strategies not common to one's field. For example, in the desensitization program occupational therapists are trained by the dental and medical staff to properly utilize the tools and simulate procedures as accurately as possible. All three models are utilized in Premier. Each approach varies depending on the patient's needs and some models may be more effective than others. Clinicians should communicate earnestly about their capabilities and concerns, and the client's needs must be central to all decisions (Schell, Gillen \& Scaffa, 2014).

Coordination of care is also essential when discussing discharge. In this program, discharge is not necessarily when a patient has "graduated" and reached the maintenance stage. Discharge can also be when a patient reaches maximum potential. Identifying a person's maximum potential requires clinical experience and judgement. This assessment requires consultation with all team members and can be a difficult topic to address with other clinicians and the caregiver. Therefore, education and consistent communication is key throughout the intervention process. Effective strategies and triggers that may escalate behavior should be reviewed with the entire team for use during future medical and dental appointments to be able to achieve the highest level of participation as possible. It is also important to note that participation and subsequent discharge from the program does not preclude a patient for joining the program again. Should circumstances change, a patient can be referred back to the desensitization program for evaluation and appropriate intervention.

Implications for Practice and Future Research

Research suggests significant autism-related health disparities, which may be additionally amplified for those individuals also who belong to disadvantaged groups, lead to more adverse physical health outcomes later in the life. This indicates that clinicians need to better understand 
the factors that promote physical health and develop interventions that maximize health and increase quality of life for people with ASD (Bishop-Fitzpatrick \& Kind, 2017).

The desensitization program has had many successful outcomes in helping patients with ASD to demonstrate less resistive behaviors and take more steps toward successfully completing medical and dental visits. The consistent collaboration, coordination, and communication amongst the patient, caregivers, therapists, and providers exemplify a standard of care that can show success, not only with the autism population, but for overall quality healthcare. Further research is needed on desensitization with this population in order to measure efficacy and develop formal training modules to expand its use. This can include more robust use of new technology, such as virtual reality, to allow practice for situations that resemble real life as accurately as possible. Technology can also help in bridging the accessibility gap regarding communication and socialization that often impedes the ability of a person with ASD to successfully participate in their daily routine.

While Premier specializes in working with patients with I/DD, and consequently its staff are better equipped to work and adapt to the unique needs that this population has, not all patients with ASD have access or use these specialty clinics and not all primary care clinics have access to behavior analysts or occupational therapists. However occupational therapists have the training to develop, test, and implement health management interventions to improve overall well-being for individuals with autism (Schwartz et al., 2019). An interdisciplinary team can help design desensitization techniques to meet the needs of health care facilities that cater to the general population but may have some patients with ASD. These can include creating a sensory friendly environment, use of additional visual supports to prepare patients for procedures, and consistent collaboration with all members of the patient's health care team. These strategies can be beneficial not only to patients with ASD, but to patients of all abilities who experience some level of anxiety during medical or dental appointments, as up to $20 \%$ of the U.S. population experience "white coat syndrome" during health care visits (Sine, 2008).

The current COVID-19 pandemic, while having a huge impact on a global scale and in its infiltration into daily life, has also highlighted the unique challenges of people with ASD. Face coverings are now the norm, but sensory tactile defensiveness can make it impossible to implement. Without the ability to tolerate wearing a face covering, people with ASD may not be able to access their community without risking their health and safety. Difficulty understanding 
the standards set forth by health officials, such as proper hand hygiene and compliance with face coverings, can negatively impact their ability to safely engage in infection control. Challenges in understanding social cues and appropriate social interactions can make the new concept of social distancing difficult to practice and therefore increase their risk of contracting the disease. Desensitization interventions such as social stories, sensory techniques, behavioral strategies, and simulations can help people with ASD implement the latest recommendations to combat COVID19.

\section{CONCLUSION}

While the most unique aspect of this program is its collaborative approach, it can prove challenging to implement. Education and staff training for the medical and dental providers is crucial for understanding and acceptance in collaboration with the patient, caregivers, and therapists. At the same time, therapists need to be trained and educated on the nuances of all the medical/dental tools and procedures in order to accurately simulate and train patients/caregivers in preparation for their real health care appointments. It also becomes challenging to appropriately generalize these skills if the patient receives medical or dental care outside of Premier or undergoes procedures outside the scope of primary care (i.e. hospitalizations, surgeries, specialty care).

The process of building trust between the patient and desensitization team may be a time intensive process but is a vital element for success of the program. The patient may become accustomed to individual clinicians and experience regression if that clinician is no longer available to work with the patient before they reach the generalization stage. This may affect the desensitization program's ability to be implemented in clinics with high turnover rates of staff or where long-term relationships between patient and clinicians are not possible.

Limitations to this study include the participant requirements of the grant. While people from all regions of New York City come to Premier HealthCare for services, only a limited number are eligible for the grant and can receive this co-treatment intervention. This limits the participant pool both for data collection and the number of beneficiaries of these services. In New York state, it is estimated that 3.8 million people have a disability, and only $7.9 \%$ of this population are able to go to the doctor alone (CDC, 2019). Ongoing needs assessments, data collection, and training is needed in order to expand services to all who may benefit from it. In addition, while the grant has a structured sequence, the implementation varies from patient to 
patient due to the unique characteristics of autism and individual patient needs. Although this allows the program and team to provide a patient-centered plan of care, it makes it challenging to development and implement standardized protocols and therefore its expansion to other settings and populations.

In conclusion, a desensitization program at Premier HealthCare, funded by a grant from the New York State Office for People with Developmental Disabilities (OPWDD), was initiated to help people with intellectual and developmental disabilities who were currently unable to tolerate routine medical and dental procedures without the use of stabilization devices or general anesthesia. The goals of the desensitization program are to reduce patient fear and anxiety related to dental and medical procedures; help patients build the coping skills needed to successfully complete dental/medical procedures; assist patients in receiving the primary care and participate in health maintenance activities; and reduce stress within families related to traumatic health care appointments. Over the past 10 years of the program, there have been a number of successes and this team continues to advocate for quality healthcare for individuals of all needs.

\section{REFERENCES}

1. Allely C., S. (2013). Pain sensitivity and observer perception of pain in individuals with autistic spectrum disorder. The Scientific World Journal, 916178. Retrieved from https://doi.org/10.1155/2013/916178

2. American Occupational Therapy Association. (2014). Occupational therapy practice framework: Domain and process (3rd ed.). American Journal of Occupational Therapy, 68 (Suppl. 1), S1-S48.

3. Armfield, J., M. (2012). The avoidance and delaying of dental visits in Australia. Australian Dental Journal, 57, 1-5.

Retrieved from https://onlinelibrary.wiley.com/doi/full/10.1111/j.1834-7819.2012.01697.x

4. Armfield, J., M., \& Heaton, L. (2013). Management of fear and anxiety in the dental clinic: A review. Aust Dent J, 58, 390-407. doi:10.1111/adj.12118

5. Bishop-Fitzpatrick, L., \& Kind, A. (2017). A Scoping Review of Health Disparities in Autism Spectrum Disorder. Journal of Autism and Developmental Disorders, 47(11), 33803391. https://doi.org/10.1007/s10803-017-3251-9 
6. Brahm C., O., Lundgren J., Carlsson S., G., Nilsson, P., Corbeil, J., \& Hagglin, C. (2012). Dentists' views on fearful patients. Problems and promises. Swedish Dental Journal, 36(2), 79-89. Retrieved from https://pubmed.ncbi.nlm.nih.gov/22876395/\#: : text=Conclusion\%3A\%20Dentists'\%20views\%20of\%20treating,do\%20not\%20appreciate $\% 20$ their\%20efforts.

7. Centers for Disease Control and Prevention. (2019). Disability and health U.S. state profile data for New York (Adults 18+ years of age). Retrieved from https://www.cdc.gov/ncbddd/disabilityandhealth/impacts/new-york.html

8. Croen, L., A., Zerbo, O., Qian, Y., Massolo, M., L., Rich, S., Sidney, S., \& Kripke, C. (2015). The health status of adults on the autism spectrum. Autism, 19(7), 814823. https://doi.org/10.1177/1362361315577517

9. Davit, C., J., Hundley, R., J., Bacic, J., D., \& Hanson, E., M. (2011). A pilot study to improve venipuncture compliance in children and adolescents with autism spectrum disorders. Journal of Developmental and Behavioral Pediatrics: JDBP, 32(7), 521525. https://doi.org/10.1097/DBP.0b013e3182245b09

10. Evans, D., W., Canavera, K., Kleinpeter, F., L., Maccubbin, E., \& Taga, K. (2005). The fears, phobias and anxieties of children with autism spectrum disorders and down syndrome: Comparisons with developmentally and chronologically age matched children. Child Psychiatry Human Development, 36(1), 3-26. doi:10.1007/s10578-004-3619-X

11. Farrugia, S., \& Hudson, J. (2006). Anxiety in adolescents with asperger syndrome: Negative thoughts, behavioral problems, and life interference. Focus on Autism and Other Developmental Disabilities, 21(1), 25-35. https://doi.org/10.1177/10883576060210010401

12. Hawkins, N., E. (1991). Bravery training: An approach to desensitizing young children to fears encountered in the hospital setting. Archives of Physical Medicine and Rehabilitation. $72,697-700$.

13. Hirvikoski T, Mittendorfer-Rutz E, Boman M, Larsson H, Lichtenstein P, \& Bölte S. (2016). Premature mortality in autism spectrum disorder. The British Journal of Psychiatry, 208 (3), $232-238$.

14. John's Hopkin's Medicine. (n.d.). Procedural sedation. Retrieved from https://www.hopkinsmedicine.org/health/treatment-tests-and-therapies/proceduralsedation 
15. Kim, J., A., Szatmari, P., Bryson, S., E., Streiner, D., L., \& Wilson, F. J. (2000). The prevalence of anxiety and mood problems among children with autism and Asperger syndrome. Autism, 4

$117-$ 132. https://doi.org/10.1177/1362361300004002002

16. Kuhaneck, H., M. \& Chisholm, E., C. (2012). Improving dental visits for individuals with autism spectrum disorders through an understanding of sensory processing. Special Care in Dentistry, 32, 229-233. doi:10.1111/j.1754-4505.2012.00283.x

17. Mouridsen, S., E., Brønnum-Hansen, H., Rich, B., \& Isager, T. (2008). Mortality and causes of death in autism spectrum disorders: An update. Autism, 12(4), 403-414.

18. Nelson, T., Chim, A., Sheller, B., McKinney, C., \& Scott, J. (2017). Predicting successful dental examinations for children with autism spectrum disorder in the context of a dental desensitization program. The Journal of the American Dental Association, 148(7), 485492. https://doi.org/10.1016/j.adaj.2017.03.015

19. Nicolaidis, C., Kripke, C. C., \& Raymaker, D. (2014). Primary care for adults on the autism spectrum. The Medical Clinics of North America, 98(5), 11691191. https://doi.org/10.1016/j.mcna.2014.06.011

20. Nicolaidis, C., Raymaker, D. M., Ashkenazy, E., McDonald, K. E., Dern, S., Baggs, A. E., Kapp, S. K., Weiner, M., \& Boisclair, W. C. (2015). Respect the way I need to communicate with you": Healthcare experiences of adults on the autism spectrum. Autism: The International Journal of Research and Practice, 19(7), 824831. https://doi.org/10.1177/1362361315576221

21. OPWDD (n.d.). Eligibility review process. Retrieved from https://opwdd.ny.gov/eligibility

22. Primeau, M., Gershon, A., \& Talbot, L., (2016). Individuals with autism spectrum disorders have equal success rate but require longer periods of systematic desensitization than control patients to complete ambulatory polysomnography. Journal of Clinical Sleep Medicine. 12(3), 357-362. doi:10.5664/jcsm.5584

23. Putallaz, F., Rubins, S., \& Schwartz, S. (1999). Mental health: Improper restraint or seclusion use places people at risk. Report prepared for United States General Accounting Office. Retrieved from https://www.gao.gov/assets/230/228149.pdf 
24. Schell, A., B., Gillen, G., \& Scaffa, M., E. (2014). Professionals, Communication, and Teamwork. In Willard \& Spackman's Occupational Therapy (pp. 457-458). Philadelphia, PA: Wolters Kluwer Health/Lippincott Williams \& Wilkins.

25. Schwartz, J., Alam, J.,Cotayo, E., Espinosa, S., Grossman, D., Herdocia, P., Hernandez, V., Lherisson, B., Lopez, K., Mejia, J., Rodriguez, C., Rodriguez, M., \& Torres, J. (2019). Understanding health management for adults with autism. American Journal of Occupational Therapy. 73(4). Retrieved from https://ajot.aota.org/article.aspx?articleid=2755160.

26. Sine, R. (2008). Beyond 'white coat syndrome': Fear of doctors and tests can hinder preventive health care. Retrieved from: http://www.webmd.com/anxietypanic/features/beyond-white-coat-syndrome.

27. Thye, M., D., Bednarz, H., M., Herringshaw, A., J., Sartin, A., B., \& Kana, R., K. (2018). The impact of atypical sensory processing on social impairments in autism spectrum disorder. Developmental Cognitive Neuroscience, 29, 51-167.

28. Tomcheck, S., D., \& Dunn, W. (2007). Sensory processing in children with and without autism: A comparative study using the Short Sensory Profile. American Journal of Occupational Therapy, 61(2), 190-200.

29. YAI. (n.d.). About YAI. Retrieved from https://www.yai.org/about

30. YAI. (n.d.). Premier HealthCare. Retrieved from https://www.yai.org/locations/healthcare

31. Zerbo, O., Massolo, M.L., Qian, Y., \& Croen, L.A. (2015). A study of physician knowledge and experience with autism in adults in a large integrated healthcare system. Journal of Autism and Developmental Disorders, 45(12), 4002-4014. 